\title{
Computed radiography exposure indices in mammography
}

\section{Liebner Koen, BMed (Hons)
Charles Herbst, PhD
William Rae, MB BCh, PhD
Department of Medical Physics, University of the Free State, Bloemfontein}

\begin{abstract}
Background. Studies indicate that computed radiography (CR) can lead to increased radiation dose to patients. It is therefore important to relate the exposure indicators provided by $\mathrm{CR}$ manufacturers to the radiation dose delivered so as to assess the radiation dose delivered to patients directly from the exposure indicators.

Aim. The aim of this study was to investigate the performance of an Agfa CR system in order to characterise the dose indicators provided by the system.

Method. The imaging plate response was characterised in terms of entrance exposure to the plate and the digital signal indicators generated by the system (SAL - scanning average level; and lgM - logarithmic median) for different beam qualities. Several exposures were performed on a mammography unit, and the digital signal, expressed in terms of SAL and lgM for each image, was correlated with the entrance exposure on a standard American College of Radiology (ACR) phantom. From this correlation, a relationship between the Agfa dose indices (SAL and $\operatorname{lgM}$ ) and the average glandular dose (AGD) in mammography could be established. An equation was derived to calculate the AGD delivered to the patient as a function of the exposure indicator, $\operatorname{lgM}$, and the $\mathrm{kV}$.

Results. The results indicated that the measured AGD at $28 \mathrm{kV}$ for a standard breast thickness during routine calibration with the ACR phantom was $1.58 \mathrm{mGy}(\operatorname{lgM}=1.99)$. This dose value lay within $1.5 \%$ of the value calculated using the derived equation for a standard Perspex thickness of $4.2 \mathrm{~cm}$ using the automatic exposure control (AEC) (1.56 mGy). The standard error in using this equation was calculated to be $8.3 \%$.
\end{abstract}

\section{Introduction}

In CR, the displayed image density is automatically adjusted by system image processing, independently of the applied dose. In fact, this is one of the key advantages of this technology; it helps to reduce the retake rate significantly, but at the same time this feature may obscure occasional or systematic under- or over-exposure. While in conventional radiography the amount of exposure is directly related to the average optical density, in CR it determines the signal-to-noise ratio (SNR), and not the image density. Higher absorbed doses result in better SNR. This is desired but, over the long term, there is a risk of a gradual drift to higher absorbed doses, as the higher exposed images tend to appear superior. ${ }^{1}$ Current clinical practice dictates that absorbed doses in radiography be minimised, while simultaneously ensuring sufficient diagnostic information in the image, and reducing the need for repeat exposures. ${ }^{2}$

The CR plate does not accumulate information about the tube current and time, $\mathrm{mAs}$ or tube potential, as $\mathrm{kVp}$ is used; it only stores energy proportional to the amount of radiation it receives. The amount of radiation that reaches the detector is influenced by the tube potential, filtration, patient thickness, exposure time, filament current and other factors. ${ }^{3}$ To quantify the response of the plate, two indices (SAL and $\operatorname{lgM}$ ) are defined by Agfa and are derived from the pixel values in the histogram of the digital image. ${ }^{4}$ These exposure indices are useful if the relationship with the air kerma is understood.

The number of pixels with the same exposure or signal level is segmented from the histogram of pixel gray-scale intensities by algorithms that remove the directly exposed area on the image and the scattered radiation to distinguish relevant from irrelevant data. The SAL is calculated as the average signal level (grey-scale value) in the image region of interest ('useful' peak in the histogram) and has a square-root relationship to the signal or the entrance kerma on the imaging plate:

$$
\begin{aligned}
& \mathrm{SAL}=\alpha \cdot \sqrt{\text { kerma }} \\
& \alpha=\alpha_{0} \sqrt{\frac{\mathrm{S}}{\mathrm{S}_{0}}}
\end{aligned}
$$

where $\alpha_{0}$ is a proportionality factor that depends on the beam quality (i.e. the radiation spectrum), ${ }^{6} \mathrm{~S}$ represents the readout speed class, and $S_{0}$ is the reference speed class equal to 200 . The square root in both equations is due to the Agfa-specific analogic filter applied to the photomultiplier output.

$\operatorname{lgM}$ is the median of $\log$ (converted pixel values) in the image region of interest (ROI) and is an indication of the dose level. It is directly related to SAL. In the case of a flat-field image, the $\operatorname{lgM}$ is specified as:

$$
\lg \mathrm{M}=2 \times \log (\mathrm{SAL})-3.9478
$$

A change of 0.3 in the numerical value of $\operatorname{lgM}$ corresponds to a change in exposure by a factor of $2 .{ }^{6}$ Photostimulable phosphor plates behave as energy-integrating detectors, and the output signal is proportional to the total deposited energy. ${ }^{5}$ To characterise the response of the plate for different exposure conditions, it is useful to correlate the output pixel value (SAL) with the entrance surface dose (ESD) and also the average glandular dose to the breast. However, the exposure level indicated by Agfa is not directly related to patient ESD, but to the light emitted during the plate read-out process. Nevertheless, when similar patient thickness and X-ray beam quality are used, the dose indicators may be related to the ESD. ${ }^{?}$

In work involving CR exposure indicators, the behaviour of the dose indicators of an Agfa CR system as a function of delivered dose has been investigated. The imaging plate response was characterised in terms of exposure and in terms of the digital signal for different beam qualities applicable in mammography using a phantom with standard thickness. From the physical characterisation of the CR system, it was possible to establish a relationship between the Agfa dose indicators and average glandular dose delivered to patients undergoing mammography examinations. An equation was derived to convert the exposure indicator lgM, to AGD. This equation could provide users with a guide to the absorbed dose delivered as a function of the Agfa indices and the tube $\mathrm{kV}$. 


\section{Material and method}

The Philips Mammo Diagnostic UC unit which we used contained a $\mathrm{Mo} / \mathrm{Mo}$ target/filter combination and was calibrated using the procedure described in the ACR manual before measurements. ${ }^{8}$ The standard ACR mammographic phantom simulated an average patient with breast thickness of $4.2 \mathrm{~cm}$ and $50 \%$ adipose $/ 50 \%$ glandular composition. An ionisation chamber and electrometer (Nero $8000 \mathrm{mAx}$ ) calibrated at mammographic X-ray beam energies (with a calibration factor traceable to international standards) were used to measure the entrance exposure in roentgens. The ionisation chamber was positioned in the X-ray field, beside the phantom, with the centre of the chamber level with the top surface of the phantom (as described by the ACR's Mammography Quality Control Manual). ${ }^{8}$ Therefore, the phantom was used to measure typical entrance exposures for an average patient and to calculate the associated average glandular dose.
Table I. Kilovoltage $(\mathrm{kV})$ range and half-value layer (HVL) in millimetres of aluminium (Al); information used to calculate average glandular dose from entrance exposure

\begin{tabular}{ccc}
\hline $\mathbf{k V}$ & HVL (mm Al) & $\begin{array}{c}\text { Glandular dose conversation } \\
\text { factor Dgn (mrad/R)[5] }\end{array}$ \\
\hline 23 & 0.27 & 135 \\
25 & 0.29 & 148 \\
26 & 0.29 & 150 \\
28 & 0.32 & 166 \\
30 & 0.33 & 173 \\
32 & 0.35 & 182 \\
34 & 0.36 & 188 \\
\hline
\end{tabular}

Table II. Summary of equation coefficients from the regression equations obtained from regression fits shown in Figs 1 and 2

\begin{tabular}{lccc}
\hline $\mathbf{k V}$ & $\begin{array}{l}\text { SAL equation coefficient } \\
\mathbf{a}\left[\mathbf{m G y} \mathbf{~ 1 0} \mathbf{~ 1 0}^{-8}\right]\end{array}$ & $\begin{array}{l}\text { IgM equation } \\
\text { coefficient } \mathbf{b}\end{array}$ & $\begin{array}{l}\text { IgM equation } \\
\text { coefficient } \mathbf{c}\end{array}$ \\
\hline 23 & 2.48 & 0.021 & 2.32 \\
25 & 2.12 & 0.019 & 2.30 \\
26 & 1.89 & 0.016 & 2.35 \\
28 & 1.82 & 0.016 & 2.31 \\
30 & 1.67 & 0.013 & 2.38 \\
32 & 1.42 & 0.012 & 2.33 \\
34 & 1.26 & 0.011 & 2.31 \\
\hline
\end{tabular}

The characterisation was performed by doing entrance exposure measurements using the manual technique, fixing the kilovoltage and varying the $\mathrm{mAs}$, and then repeating the procedure for a series of kilovoltages: 23, 25, 26, 28, 30, 32 and $34 \mathrm{kVp}$. The digital phantom images were obtained by means of an Agfa ADC compact reader using a readout class of 50.1 For each image, the SAL was evaluated as the average value of the pixels in a ROI with a dimension of approximately $10 \times 10$ $\mathrm{cm}^{2}$ centred in the middle of the plate. For each $\mathrm{kV}$, several exposures were performed, ranging from about 25 to $160 \mathrm{mAs}$. Conversion factors, depending on beam quality and filter/target combination, ${ }^{8}$ were used to convert entrance exposure to AGD. Beam quality in terms of half-value layers in mm of aluminium were measured using a standard procedure. ${ }^{8}$ Subsequently, AGD was plotted as a function of SAL and $\operatorname{lgM}$, with a minimum of 6 data points for each graph, respectively. The relationship between AGD and SAL was found to be quadratic; Microsoft Excel 2003 was used to determine this relation and optimise the fitted points. The relationship between AGD and $\lg$ M was found to be exponential. In combining the fitted equations at different beam qualities and plotting the fitted parameters against $\mathrm{kV}$, it was possible to obtain a unified equation for average glandular dose as a function of SAL/lgM and kilovoltage. The standard error in the fitted parameters was calculated.

\section{Results}

In Table I, the conversion factors for converting entrance exposure to average glandular dose are shown for each $\mathrm{kV}$ and half-value layer, respectively. As indicated in the table, the voltage ranges from $23 \mathrm{kV}$ to $34 \mathrm{kV}$, and the corresponding half-value layers varied between 0.27 and $0.36 \mathrm{~mm}$ of aluminium. This information was used to look up the average glandular dose conversion factors from the ACR manual. ${ }^{8}$

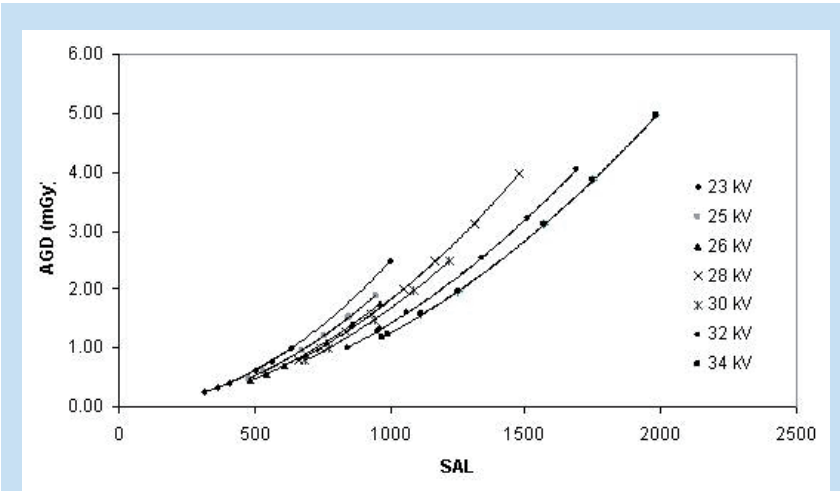

Fig. 1. AGD v. SAL on the plate at different $k V p$ values.

In Fig. 1, plots of average glandular dose versus SAL for the $\mathrm{kV}$ range are displayed. The results show that SAL, as expected from Equation 1, has a quadratic relationship to the exposure on the imaging plate. The respective regression equations in Fig. 1 can be expressed as a single equation: 


\section{REVIEW ARTICLE}

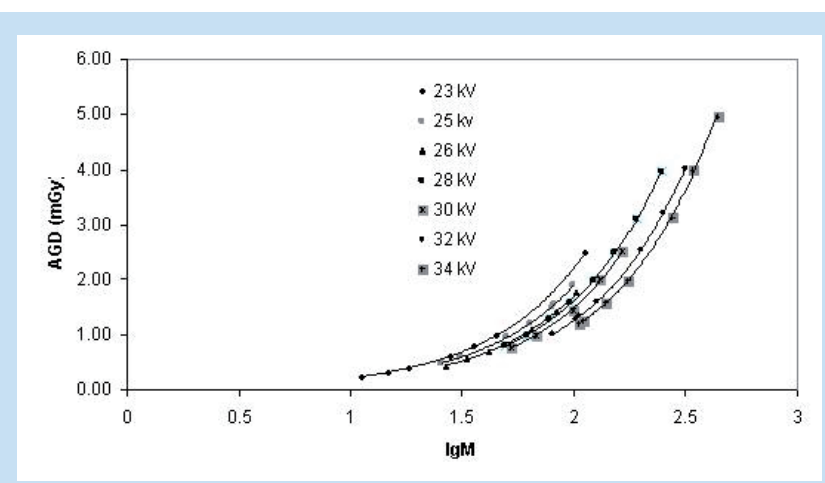

Fig. 2. $A G D$ v. IgM on the plate at different $k V p$ values.

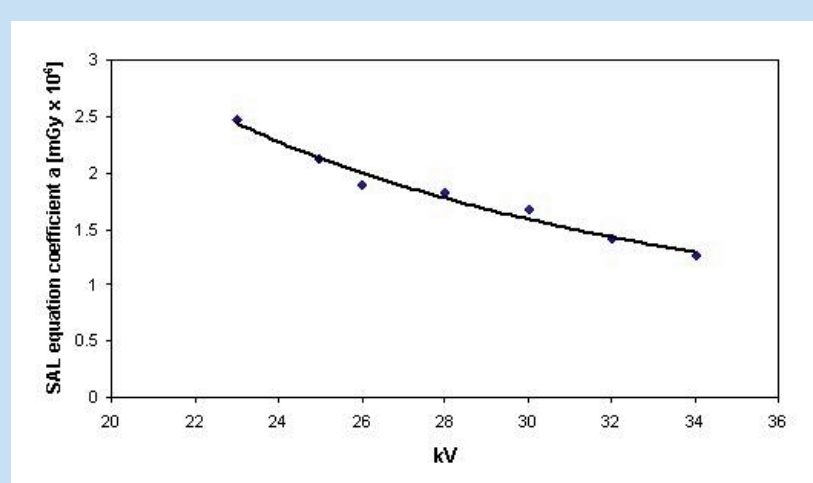

Fig. 3. A plot of the SAL equation coefficient 'a' as a function of $k V$, as indicated in Equation 3 (correlation factor $R^{2}=0.9763$ ).

$$
\operatorname{AGD}(m G y)=a \times \mathrm{SAL}^{2}
$$

where a is a parameter dependent on beam quality. The parameter values for different kilovoltages are given in Table II and are plotted in Fig. 3.

Fig. 2 shows plots of average glandular dose versus $\lg \mathrm{M}$ for different kilovoltages. The results show that $\lg M$ has an exponential relation to exposure on the imaging plate. From regression fits, the relationship between AGD and $\operatorname{lgM}$ can be expressed as:

$$
\operatorname{AGD}(\mathrm{mGy})=\mathrm{b} \times \mathrm{e}^{\mathrm{c} \times \lg M}
$$

where $b$ is a parameter dependent on beam quality and $\mathrm{c}$ is effectively a constant. These parameters are given in Table II and also plotted in Figs 4 and 5, respectively.

The relationship between coefficient 'a' and kilovoltage is as follows (regression through data points in Fig. 3):

$$
\mathrm{a}=395.71 \times 10^{-6} \times \mathrm{kV}^{-1.623}
$$

Equation 5 can be substituted into Equation 3 to calculate AGD in terms of SAL and $\mathrm{kV}$ :

$$
\mathrm{AGD}(\mathrm{mGy})=395.71 \times 10^{-6} \times \mathrm{kV}^{-1623} \times(\mathrm{SAL})^{2}
$$

The standard error in Equation 6 was calculated to be $5.1 \%$. From Fig. 4, the regression equation is as follows:

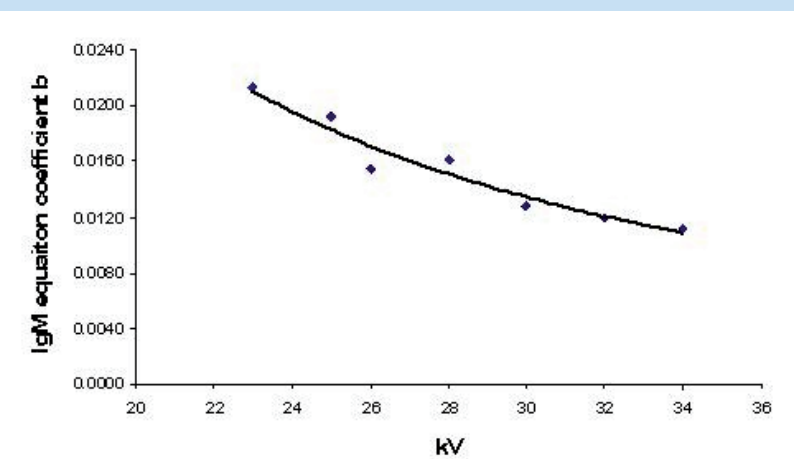

Fig. 4. IgM equation coefficient ' $b$ ' (Equation 4) as a function of $k \mathrm{~V}$ $\left(R^{2}=0.944\right)$.

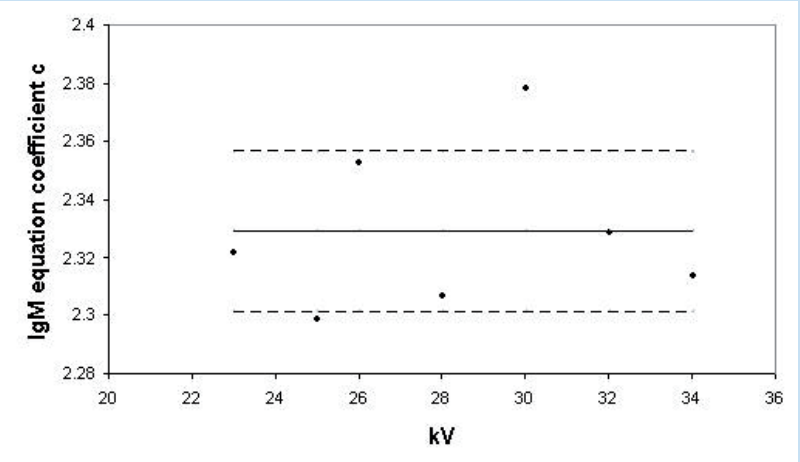

Fig. 5. IgM equation coefficient ' $c$ ' (Equation 4) as a function of kV. Average value $=2.329$.

$$
\mathrm{b}=4.1693 \times \mathrm{kV}^{-1.6865}
$$

When substituting this into Equation 4, AGD in terms of $\operatorname{lgM}$ can then be expressed as:

$$
\mathrm{AGD}(\mathrm{mGy})=4.1693 \times \mathrm{kV}^{-1.6865} \times \mathrm{e}^{2.329 \times \lg \mathrm{M}}
$$

The standard error in this equation was calculated to be $8.3 \%$. One standard deviation is shown in the figure; therefore, one would expect $67 \%$ of the values to be within the standard error indicated by the lines shown.

\section{Discussion}

The use of a standard breast phantom for entrance dose estimation does not reflect the true skin dose for larger or smaller breasts. However, this aspect does not influence the purpose of this study to investigate the relationship between average glandular dose and Agfa dose indices. Our experimental results demonstrate the dependence of exposure indices on the amount of energy deposited onto the receptor. We were forced to acquire data for individual $\mathrm{kVps}$ during separate sessions, as it was impossible to complete all the measurements in one day. In an effort to minimise systematic errors, the experimental setup was duplicated as reproducibly as possible.

The results show that a well-defined square root relationship exists between SAL and AGD, and that this relationship is dependent on 
the kilovoltage used. The dependence on kilovoltage was investigated by doing measurements at 7 different clinically relevant $\mathrm{kV}$ settings. Graphs in Fig. 1 have excellent correlation coefficients with a mean $\mathrm{R}^{2}$ value of $0.9998 \pm 0.0003$. Similarly, lgM has an exponential relationship to AGD. Once again, correlation coefficients showed a mean value of $0.9997 \pm 0.0002$ for all $\mathrm{kVs}$. The unified equations allow estimation of AGD for a standard breast thickness for any $\mathrm{kV}$ setting from the SAL or $\operatorname{lgM}$, thus providing users with guidelines for the average glandular dose delivered to patients during CR mammography.

At our centre, Agfa dose monitoring statistics ${ }^{1}$ indicated that the average $\operatorname{lgM}$ value for all the exposure types fluctuates around 2. From the measurements, an $\operatorname{lgM}$ value of 2 is equivalent to an average glandular dose of $2.48 \mathrm{mGy}(23 \mathrm{kV}) ; 1.89 \mathrm{mGy}(25 \mathrm{kV}) ; 1.58 \mathrm{mGy}(28 \mathrm{kV}) ; 1.45$ mGy $(30 \mathrm{kV}) ; 1.29 \mathrm{mGy}(32 \mathrm{kV})$; and $1.21 \mathrm{mGy}(34 \mathrm{kV})$, respectively. In using equation 8 , the AGD for $28 \mathrm{kV}$ is calculated to be $1.56 \mathrm{mGy}$ for an $\operatorname{lgM}$ value of 2 . This value is within $1.5 \%$ of the measured value (1.58 $\mathrm{mGy})$. The standard error on the equation is $8.3 \%$; therefore, estimation of dose could fluctuate around $\pm 8.3 \%$ from the true average glandular dose delivered to the patient. This result is understandable and still useful, as it is important to recognise that the exposure indices are only an estimate of the incident exposure on the imaging plate, and are not an absolute value. ${ }^{6}$ Furthermore, as the AGD is doubled, e.g. from $1 \mathrm{mGy}$ to $2 \mathrm{mGy}(28 \mathrm{kV}$ ), the corresponding increase in lgM value is 0.3 (from $\operatorname{lgM}=1.79$ to $\lg M=2.09$ ), as mentioned by other authors. ${ }^{1,2}$
One should note that the AGD is an indication of the dose to the glands measured in a phantom that is a breast equivalent and is only valid for specific $\mathrm{kV}$ and $\mathrm{mAs}$ settings; it cannot be directly applied to the human breast. The equations derived in this paper relate AGD to the SAL value received from an image. The danger exists that users may be tempted to use the SAL and the equation to calculate a particular patient dose. The change in histogram may change the SAL value and therefore the relationship.

We thank the Department of Radiology at Universitas Hospital, Bloemfontein, for accommodating our study in the department.

1. Agfa-Gevaert: ADC Compact Dose Monitoring Software User Manual. Mortsel, Belgium: Agfa-Gevaert, 1998.

2. Schaetzing R. Management of pediatric radiation dose using Agfa computed radiography. Pediatr Radiol 2004; Oct. (Suppl 1): S207-S214.

3. Willis EC. Computed radiography: a higher dose? Pediatr Radiol 2002; 32: 745-750

4. ADC Application Manual - General Radiology, 2nd ed. Mortsel, Belgium: Agfa-Gevaert, 2005.

5. Mazzocchi S, Belli S, Busoni C, et al. AEC set-up optimization with computed radiography imaging. Radiat Prot Dosimetry 2005; 117(1-3): 169-173.

6. Samei E, Seibert JA, Willis CE, et al. Acceptance testing and quality control of photostimulable phosphor imaging systems. Report of the American Association of Physicists in Medicine (AAPM), Task Group 10 (1998).

7. Sanchez Jacob R, Vano-Galvan E, Vano E, et al. Optimizing the use of computed radiography in pediatric chest imaging. J Digit Imaging 2007; September: 1-10.

8. Mammography Quality Control Manual, revised ed. Reston, USA: American College of Radiology, 1994

\section{Radiology Positions Canada}

RADIOLOGIST: SK Radiology Associates of Regina is seeking Radiologists to join 16 member sole practice in Regina. RAR serves two acute care hospitals, its two clinics as well as numerous outside clinics and rural hospitals. Canadian Royal College certification or eligibility to write exam preferred. Locum positions are also available. Regina has a booming economy, no traffic and an international airport.

Contact Larry Kliman, Business Manager tel: 306-529-3305 or e-mail larry@rarsk.com 